\title{
Binding energy constraint on matter radius and soft dipole excitations of ${ }^{22} \mathrm{C}$
}

\author{
S. N. Ershov \\ Joint Institute for Nuclear Research, RU-141980 Dubna, Russia \\ J. S. Vaagen \\ Institute of Physics and Technology, University of Bergen, N-5007 Bergen, Norway \\ M. V. Zhukov \\ Fundamental Physics, Chalmers University of Technology, S-41296 Göteborg, Sweden
}

(Received 10 July 2012; revised manuscript received 30 August 2012; published 24 September 2012)

\begin{abstract}
An unusually large value of the ${ }^{22} \mathrm{C}$ matter radius has recently been extracted from measured reaction cross sections. The giant size can be explained by a very loose binding that is, however, not known experimentally yet. Within the three-body cluster model we have explored the sensitivity of the $s$-motion-dominated ${ }^{22} \mathrm{C}$ geometry to the two-neutron separation energy. A low energy of a few tens of $\mathrm{keV}$ is required to reach the alleged experimental lower value of the matter radius, while the experimental mean radius requires an extremely tiny binding. The dependence of the ${ }^{22} \mathrm{C}$ charge radius on the two-neutron separation energy is also presented. The soft dipole mode in ${ }^{22} \mathrm{C}$ is shown to be strongly affected by the loose binding and should be studied in the process of Coulomb fragmentation.
\end{abstract}

DOI: 10.1103/PhysRevC.86.034331

\section{INTRODUCTION}

The recent claim [1] that the hitherto heaviest known Borromean two-neutron halo nucleus ${ }^{22} \mathrm{C}$ may have a giant matter radius of $5.4 \pm 0.9 \mathrm{fm}$ would be a breakthrough observation of drip-line physics if confirmed. To this end Tanaka with co-authors use a simplified three-body model of ${ }^{22} \mathrm{C}$, giving a connection between the two-neutron separation energy $S_{2 n}$ and the experimental reaction cross section. They come to the conclusion that a very small $S_{2 n} \sim 10 \mathrm{keV}$ is needed even if the two halo neutrons are situated in pure $s^{2}$ configuration.

The value of the two-neutron separation energy is not experimentally known and the last evaluation [2] assigns for ${ }^{22} \mathrm{C}$ a rather uncertain value, $S_{2 n}=0.42 \pm 0.94 \mathrm{MeV}$. Such an ambiguity has fueled discussions $[3,4]$ about ${ }^{22} \mathrm{C}$ possibly having an extremely large size.

Halo nuclei are examples of extreme clusterization into a veil of valence neutrons and a core (C). The most simple and transparent description of halo nuclear structure can be obtained within the framework of cluster models. In cluster models one assumes that the nuclear wave function of a nucleus with $A$ nucleons is factorized into a product of two parts, $\Psi\left(\mathbf{r}_{1}, \ldots, \mathbf{r}_{A}\right)=\phi\left(\mathbf{r}_{1}, \ldots, \mathbf{r}_{A_{C}}\right) \psi$. The first, $\phi\left(\mathbf{r}_{1}, \ldots, \mathbf{r}_{A_{C}}\right)$, is the core wave function describing the motion of the $A_{C}$ nucleons within the core. The second, $\psi$, describes the relative motion of the core center of mass (c.m.) and the halo nucleons, the cluster constituents.

For two-body cluster models the wave function $\psi$ depends on the binary relative coordinate $\mathbf{r}$, while for three-body cluster models this dependence includes two translational invariant Jacobi coordinates $(\mathbf{x}, \mathbf{y})$, where $\mathbf{x}$ is the relative distance between two constituents and $\mathbf{y}$ is a relative distance between the c.m. of a pair of two constituents and the third fragment. The matter root-mean-square (r.m.s.) radius of the nucleus is defined as an average of the sum of squares of intrinsic
PACS number(s): 21.10.Gv, 21.45.-v, 21.60.Gx, 27.30.+t

(defined relative to the c.m coordinate $\mathbf{R}_{\text {c.m. }}$ of nucleus $A$ ) nucleon coordinates over the nuclear wave function:

$$
\left\langle r_{m}^{2}\right\rangle_{A}=\left\langle\Psi\left(\mathbf{r}_{1}, \ldots, \mathbf{r}_{A}\right)\left|\frac{1}{A} \sum_{i=1}^{A}\left(\mathbf{r}_{i}-\mathbf{R}_{\text {c.m. }}\right)^{2}\right| \Psi\left(\mathbf{r}_{1}, \ldots, \mathbf{r}_{A}\right)\right\rangle .
$$

Calculation of this observable within cluster models leads to an expression that connects the r.m.s. matter radius of the core and an average of squares of relative distances over the wave function $\psi$. For a two-body cluster model this expression is, for example, given in Ref. [4] (the first formula in the article) and includes the average $\left\langle\psi(\mathbf{r})\left|\mathbf{r}^{2}\right| \psi(\mathbf{r})\right\rangle$, while for the three-body case the following expression is obtained [5]:

$$
\left\langle r_{m}^{2}\right\rangle_{A}=\frac{1}{A}\left\langle\psi(\mathbf{x}, \mathbf{y})\left|\rho^{2}\right| \psi(\mathbf{x}, \mathbf{y})\right\rangle+\frac{A_{C}}{A}\left\langle r_{m}^{2}\right\rangle_{C},
$$

where $\left\langle r_{m}^{2}\right\rangle_{C}$ is the squared r.m.s. matter radius of the core, while $\rho^{2}=A_{x} \mathbf{x}^{2}+A_{y} \mathbf{y}^{2}$ is the squared hyperradius and $A_{x, y}$ are the reduced masses (in units of the nucleon mass) in the corresponding Jacobian subsystem $x$ and $y$ (additional details of which are given in the next section). The appearance of the hyperradius $\rho$ in expression (2) is rather natural for three-body models where $\rho$ has a meaning similar to that of $r$ in the two-body case.

It should be noted that estimations of the matter radius of a three-body halo nucleus using a simple extension of the two-body cluster model (see Ref. [4] and references therein) to the three-body case could lead to a larger matter radius compared to that of the three-body model.

The Hamiltonian that describes the relative motion of three fragments can only be reduced to the two-body case if the neutron-neutron interaction and recoil effects in the system are neglected. Using such approximations is rather questionable if applied to Borromean systems where binary bound states for the neutron-core subsystems do not exist. 
Thus, the neutron-neutron interaction is crucial for binding the total system. In addition, the three-body kinetic energy operator always includes centrifugal barriers for hyperradial motion even in the case when all particles are moving with zero relative orbital angular momenta [5]. As a result, the two-body (point dineutron) approximations with pure $s$-state motion lead to wave functions with larger extensions than found in a true three-body treatment.

Below we study the influence of the binding energy on the matter and Coulomb radii and on the soft dipole excitations of ${ }^{22} \mathrm{C}$ in the framework of a $\left({ }^{20} \mathrm{C}+n+n\right)$ three-body model using the hyperspherical harmonic $(\mathrm{HH})$ method $[5,6]$.

\section{THE BOUND-STATE MODEL}

Let $\{i, j, k\}$ enumerate the constituents of the three-body model, let $\mathbf{r}_{1,2}$ describe the halo neutron coordinates in an arbitrary system, and let $\mathbf{r}_{C}$ denote the position of the core c.m. Jacobi coordinates for the distance $\mathbf{x}$ between the two constituents $\{j, k\}$ and $\mathbf{y}$ between the c.m. of the pair and the third fragment $\{i\}$, as well as the nuclear center-of-mass coordinate $\mathbf{R}_{\text {c.m. }}$, are given by

$$
\begin{aligned}
\mathbf{x} & =\mathbf{r}_{j}-\mathbf{r}_{k}, \quad \mathbf{y}=\mathbf{r}_{i}-\frac{A_{j} \mathbf{r}_{j}+A_{k} \mathbf{r}_{k}}{A_{j}+A_{k}}, \\
\mathbf{R}_{\mathrm{c} . \mathrm{m} .} & =\frac{1}{A}\left(A_{i} \mathbf{r}_{i}+A_{j} \mathbf{r}_{j}+A_{k} \mathbf{r}_{k}\right), \\
A & =A_{i}+A_{j}+A_{k} .
\end{aligned}
$$

Here $A_{1,2}=1, A_{C}$ and $A=\left(A_{C}+2\right)$ are masses (in units of the nucleon mass $m$ ) of halo neutrons, the core, and the halo nucleus, respectively. Below the positions of the constituents relative to the nuclear c.m. will be denoted by capital letters, $\mathbf{R}_{i}=\mathbf{r}_{i}-\mathbf{R}_{\text {c.m. }}$. For two-neutron halo nuclei only two different Jacobi coordinate systems exist since two valence neutrons are identical particles. (To simplify notation we use the same letters $\mathbf{x}, \mathbf{y}$ for different Jacobi coordinates.) One, labeled $\mathbf{T}$ (cluster representation), corresponds to the case when the relative distance $\mathbf{x}$ is between two neutrons. In the second case, called $\mathbf{Y}$ (shell-model representation), the relative distance $\mathbf{x}$ is between core $\mathrm{C}$ and one of the neutrons.

The bound-state wave function $\psi(\mathbf{x}, \mathbf{y})$ that describes relative motion of the cluster constituents is characterized by the total angular momentum $J$ and its projection $M$ on a quantized axis. Than $\psi_{J M}(\mathbf{x}, \mathbf{y})$ is the solution of the Schrödinger three-body equation

$$
\left(\hat{T}+V_{C n_{1}}+V_{C n_{2}}+V_{n_{1} n_{2}}-E\right) \psi_{J M}(\mathbf{x}, \mathbf{y})=0
$$

where $\hat{T}$ is the kinetic energy operator for relative motion of the constituents and $V_{C n_{i}}$ and $V_{n_{1} n_{2}}$ are the binary core-neutron and neutron-neutron interactions, respectively. Solutions of Eq. (4) at a negative energy $\left(E=-S_{2 n}<0\right)$ define the bound states of the halo nucleus.

For a description of the wave function $\psi_{J M}(\mathbf{x}, \mathbf{y})$ we apply the method of hyperspherical harmonics [5]. In this method the relative Jacobi coordinates $(\mathbf{x}, \mathbf{y})$ are mapped into $\left(\rho, \Omega_{5}^{\rho}\right)$, the hyperradius $\rho$, and the set of the five-dimensional angular variables $\Omega_{5}^{\rho}=\left\{\alpha_{\rho}, \hat{\mathbf{x}}, \hat{\mathbf{y}}\right\}$. The hyperradius $\rho$ and hyperangle $\alpha_{\rho}$ are introduced by the following relations:

$$
\begin{aligned}
& \rho^{2}=A_{x} x^{2}+A_{y} y^{2}, A_{x}=\frac{A_{j} A_{k}}{A_{j}+A_{k}}, A_{y}=\frac{A_{i}\left(A_{j}+A_{k}\right)}{A}, \\
& \alpha_{\rho}=\arctan \left(\sqrt{A_{x}} x / \sqrt{A_{y}} y\right), \quad 0 \leqslant \alpha_{\rho} \leqslant \pi / 2
\end{aligned}
$$

i.e., $\sqrt{A_{x}} x=\rho \sin \alpha_{\rho}$ and $\sqrt{A_{y}} y=\rho \cos \alpha_{\rho}$. Alternative and more physical definitions are

$$
\rho^{2}=\frac{1}{A} \sum_{i>j=1,2, C} A_{i} A_{j}\left(\mathbf{r}_{i}-\mathbf{r}_{j}\right)^{2}=\sum_{i=1,2, C} A_{i} \mathbf{R}_{i}^{2} .
$$

The hyperradius $\rho$ reflects the size of the three-body system or more exactly the moment of inertia and is the same in any system of Jacobi coordinates. The angular variables $\Omega_{5}^{\rho}$ depend on the selection of the Jacobi coordinate system.

The wave function $\psi_{J M}$ is decomposed [5] into hyperspherical harmonics as

$$
\psi_{J M}(\mathbf{x}, \mathbf{y})=\frac{1}{\rho^{5 / 2}} \sum_{K \gamma} \chi_{K \gamma}^{J}(\rho)\left[\mathcal{Y}_{K L}^{l_{x} l_{y}}\left(\Omega_{5}^{\rho}\right) \otimes \chi_{S}\right]_{J M},
$$

which provide a complete orthogonal set on the fivedimensional sphere of unit hyperradius. Here, $K$ is the hypermoment, $\gamma=\left\{S, L, l_{x}, l_{y}\right\}$ is an abbreviation for a set of quantum numbers, which characterizes the relative motion of the three fragments (where $l_{x}$ and $l_{y}$ are the relative orbital angular momenta for $x$ and $y$ motions and $L$ is the total orbital moment), and $\chi_{S M_{S}}$ is the spin function of the halo neutron pair with spin $S$ and projection $M_{S}$. The five-dimensional hyperharmonic $\mathcal{Y}_{K L M_{L}}^{l_{x} l_{y}}\left(\Omega_{5}^{\rho}\right)$ has the explicit form

$$
\mathcal{Y}_{K L M}^{l_{x} l_{y}}\left(\Omega_{5}^{\rho}\right)=\psi_{K}^{l_{x} l_{y}}\left(\alpha_{\rho}\right)\left[Y_{l_{x}}(\hat{\mathbf{x}}) \otimes Y_{l_{y}}(\hat{\mathbf{y}})\right]_{L M},
$$

with the hyperangular function $\psi_{K}^{l_{x} l_{y}}\left(\alpha_{\rho}\right)$ defined in Ref. [5]. Inserting decomposition (7) into the Schrödinger equation (4) and projecting out the hyperharmonics $\mathcal{Y}_{K L M}^{l_{x} l_{y}}\left(\Omega_{5}^{\rho}\right)$, we can get the set of coupled $K$-harmonic equations for the hyperradial part $\chi_{K \gamma}^{J}(\rho)$ :

$$
\begin{gathered}
\left(-\frac{\hbar^{2}}{2 m}\left[\frac{d^{2}}{d \rho^{2}}-\frac{\mathcal{L}(\mathcal{L}+1)}{\rho^{2}}\right]-E\right) \chi_{K \gamma}^{J}(\rho) \\
=-\sum_{K^{\prime} \gamma^{\prime}} V_{K \gamma, K^{\prime} \gamma^{\prime}}^{J}(\rho) \chi_{K^{\prime} \gamma^{\prime}}^{J}(\rho),
\end{gathered}
$$

where $\mathcal{L}=K+3 / 2$ and the matrix elements are generated from binary interactions in the following way:

$$
\begin{aligned}
V_{K \gamma, K^{\prime} \gamma^{\prime}}^{J}(\rho)= & \left\langle\left[\mathcal{Y}_{K L}^{l_{x} l_{y}}\left(\Omega_{5}^{\rho}\right) \otimes \chi_{S}\right]_{J M}\right| V_{C n_{1}}+V_{C n_{2}} \\
& +V_{n_{1} n_{2}}\left|\left[\mathcal{Y}_{K^{\prime} L^{\prime}}^{l^{\prime} l_{y}^{\prime}}\left(\Omega_{5}^{\rho}\right) \otimes \chi_{S^{\prime}}\right]_{J M}\right\rangle .
\end{aligned}
$$

The asymptotic behavior for a Borromean three-body halo system in the hyperradius is similar to that of a two-body system in the binary separation, and for the bound state they are determined by the binding energy:

$$
\chi_{K \gamma}^{J}(\rho \rightarrow 0) \sim \rho^{\mathcal{L}+1}, \quad \chi_{K \gamma}^{J}(\rho \rightarrow \infty) \sim \exp [-\kappa \rho],
$$

where $\kappa=\sqrt{2 m|E| / \hbar^{2}}$. The automatic incorporation of the correct three-body asymptotic of bound-state wave functions 
for Borromean systems is one of the advantages of the hyperspherical harmonic method.

Usually the system of hyperradial equations (9) is solved in the $\mathbf{T}$ system of Jacobi coordinates since the symmetry under permutation of two valence neutrons reduces the number of allowed configurations and leads to smaller dimension of coupled equations compared with the case of the $\mathbf{Y}$ system where permutation symmetry does not appear explicitly. A unitary transformation between representations in different Jacobi systems can be done by using the Raynal-Revai coefficients [7].

\section{DISCUSSION}

\section{A. Bound-state properties}

Within a cluster three-body $\left({ }^{20} \mathrm{C}+n+n\right)$ model the bound-state properties of ${ }^{22} \mathrm{C}$ are defined by the intercluster potentials. The main problem in selecting binary potentials between the constituents is the absence of experimental information about the neutron-core system, beyond the fact that a bound state of ${ }^{21} \mathrm{C}$ does not exist. Within the shell-model picture this implies that the $1 s_{1 / 2}$ orbit is unbound. In the single available realistic three-body calculation of the ${ }^{22} \mathrm{C}$ nuclear structure [8], deep potentials that also support extra orbits including $0 s_{1 / 2}$ were used, and these forbidden states were projected out in the process of wave function calculations. These calculations, by Horiuchi and Suzuki, have been done within the stochastic variational method [9,10], which is well suited for solving three-body problems. The authors got (using different $n-{ }^{20} \mathrm{C}$ interactions) $S_{2 n}=0.388-0.573 \mathrm{MeV}$ and, correspondingly, a ${ }^{22} \mathrm{C}$ r.m.s. matter radius of $r_{m}=$ 3.74-3.58 fm (using a $2.98 \mathrm{fm}[8]$ r.m.s. radius of the ${ }^{20} \mathrm{C}$ core). There are several important findings in this paper: Noncentral forces give negligible contributions to the results, the $\left(s_{1 / 2}\right)^{2}$ configuration is predominant, many other components add only small admixtures, and the ground state of ${ }^{22} \mathrm{C}$ consists almost entirely of configurations with total spin of halo neutrons $S=0$ (with weight more then 98\%), emphasizing the halo structure of this nucleus. This underpins our model assumptions.

Since our aim is to explore principal relationships between binding energies and matter radii of ${ }^{22} \mathrm{C}$, we use a simpler approach and apply shallow potentials that do not support bound states. Using the findings of [8], we neglect noncentral forces and wave function components with total $\operatorname{spin} S=1$. We use a central neutron-core potential of the Woods-Saxon shape, with diffuseness $a=0.6 \mathrm{fm}$ and radius $R=3.5 \mathrm{fm}$, the same geometry as in Ref. [8]. The potential depth is varied from the deepest possible (that does not support neutron-core binding) to more shallow ones. The neutron-neutron potential has a Gaussian shape (depth $V=-31 \mathrm{MeV}$ and radius $R=1.8 \mathrm{fm}$ ) and reproduces the basic properties of the $n-n$ interaction [11]. The number of coupled equations in Eq. (9) depends on the number of hypermoments $K$ included in the decomposition; all combinations of orbital angular momenta $\left(l_{x}, l_{y}\right)$ possible for given $K$ are included and only even values of $K$ are allowed for positive-parity states.

The cutoff $K_{\max }$ in hyperspherical harmonics included in the wave function decomposition (7) provides a convenient

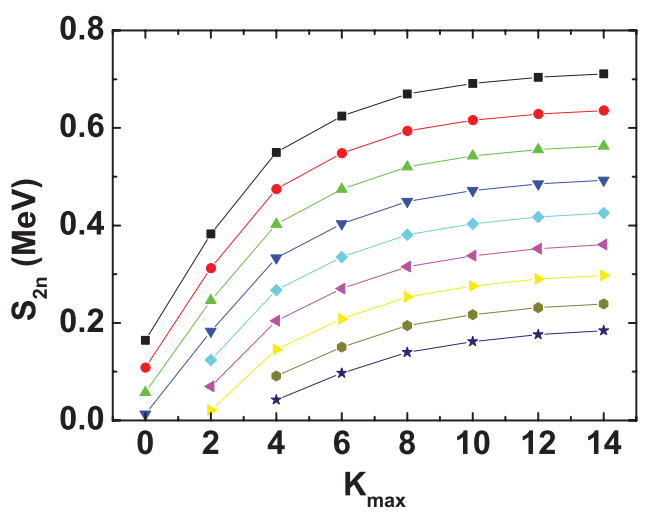

FIG. 1. (Color online) Convergence of two-neutron separation energy calculations relative to the cutoff in hypermoments, $K_{\max }$. The curves correspond to calculations with neutron-core potential with depth ranging from $-4.1 \mathrm{MeV}$ (the upper one) to $-3.3 \mathrm{MeV}$ (the lowest one) with steps of $0.1 \mathrm{MeV}$, with none giving rise to bound neutron-core states.

way to control the convergence of calculations. Figure 1 shows the convergence of the ${ }^{22} \mathrm{C}$ ground-state binding with the number of hypermoments included in the decomposition, expressed in terms of $K_{\max }$. Different curves correspond to calculations with different depths of neutron-core potentials given in the caption. Calculations with hypermoment up to $K_{\max }=14$ are close to convergence, independent of the potential depth. Thus all exploratory calculations of the bound state given below will correspond to bases with $K_{\max }=14$ leading to 20 coupled hyperradial Schrödinger equations in the Jacobi $\mathbf{T}$ system.

Figure 2 shows the calculated dependence of the ${ }^{22} \mathrm{C}$ matter radius (2) on the value of the two-neutron separation energy. The experimental value of $2.98 \mathrm{fm}$ extracted from reaction

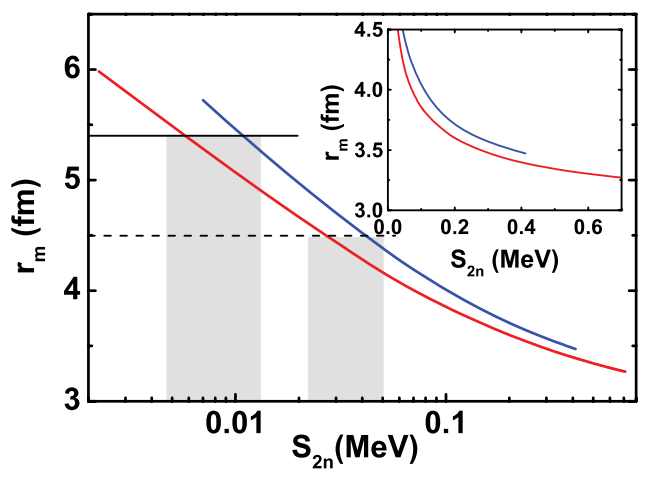

FIG. 2. (Color online) The dependence of the ${ }^{22} \mathrm{C}$ r.m.s. matter radius on the two-neutron separation energy (on a logarithmic scale). The lower and upper curves correspond to calculations with the radius of the neutron-core potential equal to 3.5 and $4.5 \mathrm{fm}$, respectively. The horizontal solid and dashed lines are the mean value and lower boundary of the experimental matter radius [1], respectively. Bars show theoretical estimations of the $S_{2 n}$ energy at which the experimental mean value and lower boundary of the matter radius can be obtained. The insert shows the same curves, but on a linear energy scale. 
cross sections [12] was used as the ${ }^{20} \mathrm{C}$ core matter radius. The lower curve corresponds to calculations with the geometry mentioned above. The values of matter radii calculated here are slightly smaller than the ones reported in Ref. [8] at corresponding energies. One of the reasons for this may be that at the ground-state energy the width of deep potentials assumed in Ref. [8] is essentially larger than for our shallow potentials.

To check the sensitivity of the ${ }^{22} \mathrm{C}$ r.m.s radius to the potential geometry we also performed calculations (the upper curve in Fig. 2) with a neutron-core potential having a $1 \mathrm{fm}$ increased radius of $4.5 \mathrm{fm}$. As expected, a broader potential leads to larger radii at the same binding energy. The upper curve starts from ground-state energies of about $400 \mathrm{keV}$. Continuation of this curve to larger binding energies requires larger depths of the core-neutron potential and leads to the appearance of two-body bound states. As mentioned above, such potentials were excluded from consideration.

Concerning the general behavior of the ${ }^{22} \mathrm{C}$ matter radius with binding energy, we notice that this dependence (see the insert in Fig. 2) is close to linear at larger separation energies, becoming strongly nonlinear at smaller energies and diverging in the vicinity of the breakup threshold. The main frame of Fig. 2 shows the same curves versus a logarithmic scale of energy. The figure compares calculations with the values of the ${ }^{22} \mathrm{C}$ matter radius tentatively extracted [1] from experimental cross sections. Bars show that even the lower experimental value of this radius would require quite small binding energy of a few tens of $\mathrm{keV}$, diminishing to a very tiny binding for the suggested ${ }^{22} \mathrm{C}$ experimental value.

Table I lists different computed radii that characterize the geometrical properties of the ${ }^{22} \mathrm{C}$ nucleus for a set of two-neutron separation energies. Here, $r_{m}$ and $\bar{\rho}$ are the ${ }^{22} \mathrm{C}$ r.m.s. matter radius and average value of the hyperradius, respectively. $R_{C}$ and $R_{n}$ are the average core and neutron distances, respectively, from the nuclear c.m., while $r_{n n}$ and $r_{C n}$ are the relative distances between the halo constituents. The last two columns contain distances between the c.m. of a constituent pair and the third fragment. All these distances increase with decreasing binding energy, thus showing the general swelling of the halo nucleus.

Table II gives main partial weights of $\mathrm{HH}$ components of the ${ }^{22} \mathrm{C}$ ground state as a function of the two-neutron separation energy $S_{2 n}$ in the $\mathbf{T}$ and $\mathbf{Y}$ Jacobi coordinate systems. At all energies we observe the strong dominance of the $K=0$ component with weight larger than $90 \%$ and that this weight increases only slowly when separation energy becomes smaller.
TABLE II. Partial weights (in percent) of the main components of the ${ }^{22} \mathrm{C}$ ground-state $\mathrm{HH}$ wave function for different two-neutron separation energies $S_{2 n}$. The results correspond to calculations with the radius of the core-neutron potential equal to $3.5 \mathrm{fm}$.

\begin{tabular}{llllll}
\hline \hline $\begin{array}{l}S_{2 n}(\mathrm{MeV}) \\
\left(K, l_{x}=l_{y}\right)\end{array}$ & 0.40 & 0.20 & 0.10 & 0.05 & 0.01 \\
\hline \multicolumn{5}{c}{ T coordinate system } \\
$(0,0)$ & 93.7 & 93.8 & 94.0 & 94.3 & 94.8 \\
$(2,0)$ & 4.10 & 3.89 & 3.64 & 3.38 & 2.94 \\
$(4,2)$ & 0.69 & 0.76 & 0.81 & 0.84 & 0.88 \\
$(4,0)$ & 0.75 & 0.72 & 0.67 & 0.62 & 0.53 \\
$(6,2)$ & 0.0010 & 0.0013 & 0.0015 & 0.0017 & 0.0020 \\
$(6,0)$ & 0.39 & 0.41 & 0.41 & 0.40 & 0.37 \\
& \multicolumn{5}{c}{ Y coordinate system } \\
$(0,0)$ & 93.7 & 93.8 & 94.0 & 94.3 & 94.8 \\
$(2,1)$ & 4.09 & 3.88 & 3.63 & 3.38 & 2.93 \\
$(2,0)$ & 0.0093 & 0.0088 & 0.0082 & 0.0077 & 0.0067 \\
$(4,2)$ & 1.01 & 1.00 & 0.96 & 0.91 & 0.82 \\
$(4,1)$ & 0.0030 & 0.0028 & 0.0027 & 0.0026 & 0.0025 \\
$(4,0)$ & 0.43 & 0.48 & 0.51 & 0.54 & 0.59 \\
\hline \hline
\end{tabular}

Additional insight into the calculations can be obtained by studying the dependence (shown in Fig. 3) of the matter radius on the number of hypermoments in the wave function decomposition. At larger separation energies the convergence of $r_{m}$ is rather fast, but with decreasing binding it becomes increasingly slow and may demand inclusion of more hyperharmonics at very loose binding. For example, at energy $S_{2 n} \simeq 10 \mathrm{keV}, r_{m}$ increases by $2 \%$ when $K_{\max }$ changes from 10 to 14 . Since the Borromean wave functions have asymptotic exponential decay in the hyperradius, governed by $\sqrt{S_{2 n}}$ [see Eq. (11)], we note that, for weakly bound states, the wave functions decay very slowly with hyperradius $\rho$ and calculations of matter radii can require knowledge of wave functions to very large distances of order $500 \mathrm{fm}$ or more.

Sometimes it is instructive to know the scattering length of the neutron-core potential that is used in the three-body calculations. The dashed line in Fig. 3 shows the absolute value of the scattering length for potentials used in calculations with $K_{\max }=14$. The requirement of absence of bound states restricts the depth of a potential at fixed geometry and accesses only negative values of the scattering length. The deepest allowed potentials have largest negative scattering length (of the order of $-1000 \mathrm{fm}$ ) and lead to the most bound states. Reducing the potential depth decreases binding and the (absolute)

TABLE I. Dependence of the ${ }^{22} \mathrm{C}$ geometrical properties on the separation energy $S_{2 n}$ given in MeV. Radii are given in units of femtometers. The results correspond to calculations with the radius of the core-neutron potential equal to 3.5 fm.

\begin{tabular}{rcrcccrrr}
\hline \hline$S_{2 n}$ & $r_{m}$ & \multicolumn{1}{c}{$\bar{\rho}$} & $R_{C}$ & \multicolumn{1}{c}{$R_{n}$} & $r_{n n}$ & \multicolumn{1}{c}{$r_{C n}$} & $r_{C(n n)}$ & $r_{(C n) n}$ \\
\hline 0.40 & 3.40 & 8.73 & 0.45 & 6.01 & 7.87 & 6.36 & 4.99 & 6.29 \\
0.20 & 3.60 & 10.36 & 0.53 & 7.13 & 9.45 & 7.54 & 5.87 & 7.47 \\
0.10 & 3.85 & 12.19 & 0.62 & 8.39 & 11.27 & 8.86 & 6.84 & 8.79 \\
0.05 & 4.16 & 14.23 & 0.72 & 9.80 & 13.32 & 10.34 & 7.91 & 10.27 \\
0.01 & 5.01 & 19.36 & 0.96 & 13.35 & 18.52 & 14.05 & 10.57 & 13.98 \\
\hline \hline
\end{tabular}




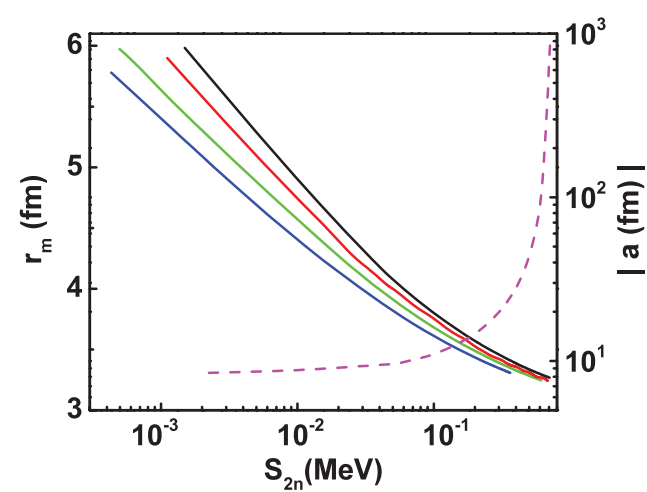

FIG. 3. (Color online) Dependence of the ${ }^{22} \mathrm{C}$ r.m.s. matter radius (left axis) on the number of hypermoments. The solid lines from lower to upper correspond to calculations with cutoff $K_{\max }=2,6$, 10 , and 14 , respectively. The dashed line shows the absolute value of the scattering length (right axis, logarithmic) of the neutron-core potential used for calculations with $K_{\max }=14$. All results correspond to calculations with the radius of the core-neutron potential equal to $3.5 \mathrm{fm}$.

scattering length is decreased. At weak binding energy rather small variation of the potential depth is enough to change the binding, leading to rather small variations in scattering length: the corresponding curve in Fig. 3 becomes flat.

For the $s d$-shell nuclei like the heavy carbon isotopes the competition between the $s$ and $d$ motions is quite important. We used in our calculations the same core-neutron potential for all partial waves. It may be that a more refined description will require different potentials for different partial waves. To check the sensitivity to this aspect of dynamics some exploratory calculations (in the $\mathbf{T}$ system of Jacobi coordinate) with an increased role of $d$-wave interaction were performed. Thus we increased the depth of the neutron-core potential for $d$-wave motion. Simultaneously the potential depth for other partial waves was slightly decreased to compensate for the enhanced $d$-wave attraction and thus avoid change in the ${ }^{22} \mathrm{C}$ binding energy. This procedure allowed us to decrease the role of $s$ motion and increase the weight of $d$-wave components of the ground-state wave function. The matter radius of ${ }^{22} \mathrm{C}$ now becomes always smaller compared with that from previous calculations. This again confirms the dominant role of the $s$ motion for obtaining extreme sizes of halo nuclei.

The other interesting observable, tightly connected with the spatial extension of the ground-state wave function, is the charge radius for pointlike protons:

$$
\left\langle r_{\mathrm{ch}}^{2}\right\rangle_{A}=\left\langle\Psi\left(\mathbf{r}_{1}, \ldots, \mathbf{r}_{A}\right)\left|\frac{1}{Z} \sum_{i=1}^{Z} \mathbf{R}_{i}^{2}\right| \Psi\left(\mathbf{r}_{1}, \ldots, \mathbf{r}_{A}\right)\right\rangle,
$$

where summation is over all protons of the nucleus. In stable nuclei the charge and matter sizes are close to each other, whereas in neutron halo nuclei they are strikingly different, with all protons being situated in the core. Within the three-body model such a concentration of charge leads to a very simple relation $[5,13,14]$ between the charge radii of the nucleus and core:

$$
\left\langle r_{\mathrm{ch}}^{2}\right\rangle_{A}=\left\langle r_{\mathrm{ch}}^{2}\right\rangle_{C}+\left\langle\psi_{J M}(\mathbf{x}, \mathbf{y})\left|\mathbf{R}_{C}^{2}\right| \psi_{J M}(\mathbf{x}, \mathbf{y})\right\rangle,
$$

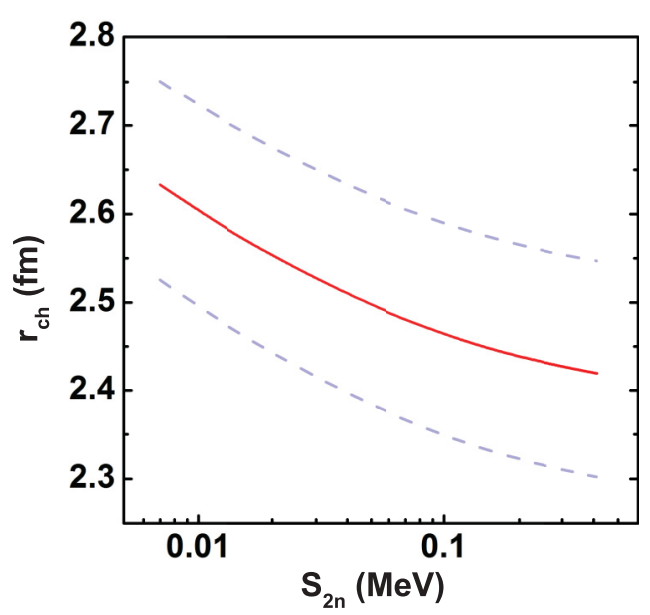

FIG. 4. (Color online) Dependence of the ${ }^{22} \mathrm{C}$ charge radius for pointlike protons on the two-neutron separation energy (on a logarithmic scale) of the ground state. The lower, middle, and upper curves correspond to calculations with the ${ }^{20} \mathrm{C}$ core charge radius equal to $2.25,2.37$, and $2.50 \mathrm{fm}$, respectively. All results are given for the core-neutron potential with a radius of $4.5 \mathrm{fm}$.

where $\left\langle r_{\mathrm{ch}}^{2}\right\rangle_{C}$ is the square of the charge radius for pointlike protons in the core, and the second term is the square of the average distance of the core c.m. from the nuclear c.m. For fixed $\rho$ value, the distance of the core to the nucleus $(A)$ c.m. defines the distances of halo neutrons to the c.m. and the size of the three-body system can be deduced. The core motion around the c.m. of the nucleus leads to a situation where the core occupies an enlarged space compared to its own size. For an external viewer it may appear as a swelling of the core. Figure 4 shows the dependence of the ${ }^{22} \mathrm{C}$ point-proton charge radius on the binding energy, for different values of the ${ }^{20} \mathrm{C}$ charge radius that are compatible with the mean-field calculations performed in Ref. [15]. The charge radii are significantly smaller (as expected) than the matter radii, but they are also growing when binding becomes weaker. Qualitatively this behavior is similar to the behavior of matter radii, but quantitatively the rate for the charge radius increase is smaller than that for the matter radius. Thus with decreasing binding, the difference between the charge and matter radii becomes more and more pronounced.

\section{B. Soft dipole excitations}

Characteristics of nuclear halos are revealed not only in the specific structure of the ground state (loosely bound, abnormal spatial extension with extreme clusterization) but also in low-energy excitations above the breakup threshold where a concentration of transition strength is observed. The most spectacular is the appearance of a soft dipole excitation mode that dominates the electromagnetic dissociation (EMD) cross section. In stable nuclei all dipole excitations are usually concentrated in the giant dipole resonance that corresponds to high-frequency collective proton-neutron vibrations. In neutron halo nuclei all charges are concentrated in the core; thus the charge and matter c.m. do not coincide and lowfrequency dipole oscillations of the halo neutrons against the core may easily be excited. Historically the large EMD cross 
sections for ${ }^{11} \mathrm{Li}$ incident on heavy targets were predicted [16] by assuming that the ${ }^{11} \mathrm{Li}$ nucleus is composed of a ${ }^{9} \mathrm{Li}$ core and a dineutron, and this subsequently confirmed experimentally (see, for example, Ref. [17]). Existence of a new low-lying dipole resonance mode (the so-called soft dipole mode) in such systems was suggested by Ikeda [18]. It should be noted that, at least for the lightest Borromean halo nuclei ${ }^{6} \mathrm{He}$ and ${ }^{11} \mathrm{Li}$, the low-lying dipole excitation mode is not a resonant one but a more general three-body low-lying continuum response [19].

The electric dipole excitations describe transitions from ground to continuum states by action of the dipole operator. For a two-neutron halo nucleus with inert core the $E 1$ excitations can be expressed by

$$
\begin{aligned}
\frac{d B(E 1)}{d E_{T}}= & \frac{(e Z)^{2}}{2 J+1} \sum_{\nu_{1}, \nu_{2}, M, \mu} \int d \mathbf{k}_{x} d \mathbf{k}_{y} \delta\left(E-E_{T}\right) \\
& \times \mid\left\langle\psi_{v_{1}, \nu_{2}}^{(-)}\left(\mathbf{k}_{x}, \mathbf{k}_{y} ; \mathbf{x}, \mathbf{y}\right)\right| R_{C} Y_{1 \mu}\left(\hat{\mathbf{R}}_{C}\right) \\
& \times\left.\left|\psi_{J M}(\mathbf{x}, \mathbf{y})\right\rangle\right|^{2},
\end{aligned}
$$

where $\psi_{\nu_{1}, \nu_{2}}^{(-)}\left(\mathbf{k}_{x}, \mathbf{k}_{y} ; \mathbf{x}, \mathbf{y}\right)$ represents the three-body continuum wave functions with ingoing wave boundary conditions that describe the relative motion of the fragments. This motion is characterized by the Jacobi linear momenta $\mathbf{k}_{x}$ and $\mathbf{k}_{y}$ conjugated to the Jacobi coordinates $\mathbf{x}$ and $\mathbf{y}$ (3). Here $E_{T}=\left(\hbar^{2} / 2 m\right)\left(\mathbf{k}_{x}^{2} / A_{x}+\mathbf{k}_{y}^{2} / A_{y}\right)=\left(\hbar^{2} / 2 m\right) \kappa_{T}^{2}$ is the total continuum energy above the breakup threshold, and $v_{1,2}$ are spin projections of halo neutrons and $\mu=( \pm 1,0)$. The continuum wave function can be decomposed in the five-dimensional hyperspherical harmonics in $\left(\Omega_{5}^{K_{T}}\right)$ defined in the space of linear Jacobian momenta [20]:

$$
\begin{aligned}
& \psi_{\nu_{1}, \nu_{2}}^{(-)}\left(\mathbf{k}_{x}, \mathbf{k}_{y} ; \mathbf{x}, \mathbf{y}\right) \\
& =\sum_{K_{f}, \gamma_{f}, M_{S_{f}}, M_{L_{f}}, M_{J_{f}}} l^{K_{f}}\left(\frac{1}{2} v_{1} \frac{1}{2} v_{2} \mid S_{f} M_{S_{f}}\right) \\
& \quad \times\left(L_{f} M_{L_{f}} S_{f} M_{S_{f}} \mid J_{f} M_{f}\right) \mathcal{Y}_{K_{f} L_{f} M_{L_{f}}^{f} l_{y}^{f} *} \\
& \quad \times\left(\Omega_{5}^{\kappa_{T}}\right) \psi_{K_{f} \gamma_{f} J_{f} M_{f}}\left(\kappa_{T}, \mathbf{x}, \mathbf{y}\right),
\end{aligned}
$$

where the spatial part $\psi_{K_{f} \gamma_{f} J_{f} M_{f}}\left(\kappa_{T}, \mathbf{x}, \mathbf{y}\right)$ only depends on spatial coordinates $(\mathbf{x}, \mathbf{y})$ and has a hyperspherical decomposition similar to Eq. (7) for the bound-state wave function $\psi_{J M}(\mathbf{x}, \mathbf{y})$ and can be obtained from the solution of the system of coupled equations (9) with boundary conditions appropriate for continuum states. Due to the selection rules for the dipole operator only the dipole excitations with $J_{f}^{\pi_{f}}=1^{-}$(with only odd values of $K_{f}$ being allowed) will give contributions to $d B(E 1) / d E_{T}$ for a ground state with quantum numbers $J^{\pi}=0^{+}$.

The distribution of soft dipole transitions is very sensitive to the ground-state binding and the total strength is directly restricted (within the three-body cluster model) by the displacement $\sqrt{\left\langle\left|\mathbf{R}_{C}^{2}\right|\right\rangle}$ of the charge c.m. against the matter c.m. [21]:

$$
\int d E_{T} \frac{d B(E 1)}{d E_{T}}=\frac{3}{4 \pi}(e Z)^{2}\left\langle\psi_{J M}(\mathbf{x}, \mathbf{y})\left|\mathbf{R}_{C}^{2}\right| \psi_{J M}(\mathbf{x}, \mathbf{y})\right\rangle .
$$

Formula (16) is the cluster non-energy-weighted sum rule (CNEWSR) for dipole excitations and values of the core displacement for the ${ }^{22} \mathrm{C}$ ground state for different separation energies given in the column $R_{C}$ of Table I. Another useful relation is the cluster energy-weighted sum rule (CEWSR) [22] for soft dipole excitations:

$$
\int d E_{T} E_{T} \frac{d B(E 1)}{d E_{T}}=e^{2} \frac{9}{4 \pi} \frac{\hbar^{2}}{2 m} \frac{2 Z^{2}}{A A_{C}} .
$$

Sum rule (17) is a part of the total energy-weighted sum rule [with $N Z / A$ replacing $2 Z^{2} /\left(A A_{C}\right)$ ] and accounts for dipole excitations due to only relative motion of the core and halo neutrons, thus excluding dipole excitations within the core.

The convergence of the $d B(E 1) / d E_{T}$ calculations for different number of hypermoments $K_{f}$ included in the decomposition (15) of the continuum wave function is shown in Fig. 5 for two of the cases given in Table I for the ${ }^{22} \mathrm{C}$ bound state, those with $S_{2 n}=400 \mathrm{keV}$ and $50 \mathrm{keV}$. Within the hyperspherical harmonics method the electric dipole transitions can only couple the spatial components of bound and continuum states with hypermoments that differ by one. The dominance of the $K=0$ bound-state component (see, for example, Table II) leads in the dipole strength functions to the prevalence of transitions with $K_{f}=1$. Inclusion of additional continuum components increases the height of dipole peaks (about $5 \%$ when $K_{f, \max }$ increases from 9 to 13 while CNEWSR is changed by $0.3 \%$ ). The rate of convergence depends on

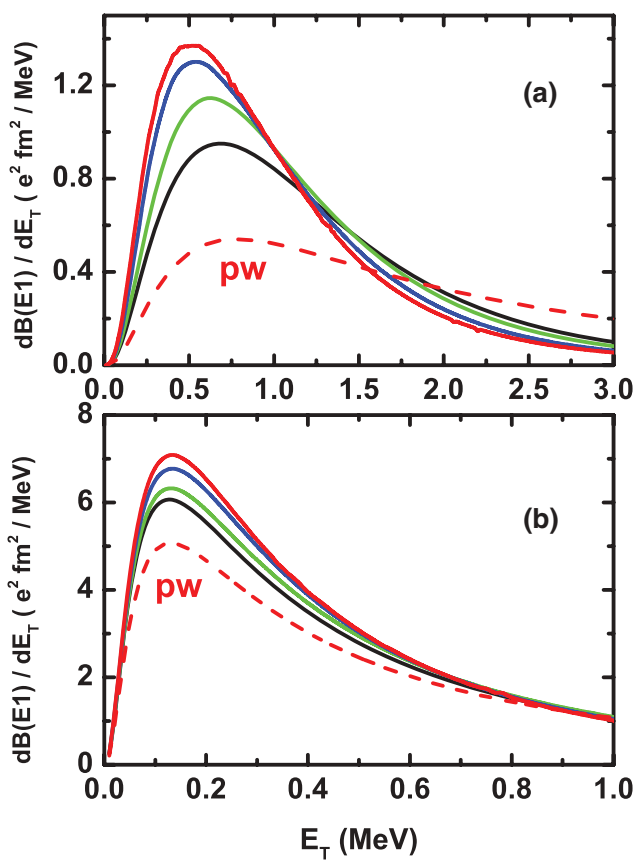

FIG. 5. (Color online) Calculations for ${ }^{22} \mathrm{C}$ dipole strength function distributions over total continuum energy $E_{T}$ above the breakup threshold. Calculations for ${ }^{22} \mathrm{C}$ two-neutron separation energies $S_{2 n}=400 \mathrm{keV}$ (a) and $50 \mathrm{keV}$ (b) are shown. The solid lines from upper to lower correspond to calculations of the dipole continuum wave functions with $K_{f \text {, max }}=13,9,5$, and 1 , respectively. The dashed lines show distributions for three-body plane wave (no FSI) continuum states. 
TABLE III. The cluster non-energy-weighted sum rule of the ${ }^{22} \mathrm{C}$ dipole strength functions calculated for different separation energies $S_{2 n}$ of the ground state. The sum rule is given for two ranges of the total continuum energy $E_{T}$ and measured in percent of the computed (16) total sum rule (the column CNEWSR). Columns marked FSI (PW) show results of calculations with (without) inclusion of the final-state interactions. The column labeled CEWSR gives the calculated cluster energy-weighted sum rule measured in percent of the total value (17) of $2.43\left(e^{2} \mathrm{fm}^{2} \mathrm{MeV}\right)$.

\begin{tabular}{|c|c|c|c|c|c|c|}
\hline $\begin{array}{l}S_{2 n} \\
(\mathrm{MeV})\end{array}$ & \multicolumn{2}{|c|}{$E_{T} \leqslant 5 \mathrm{MeV}$} & \multicolumn{3}{|c|}{$E_{T} \leqslant 10 \mathrm{MeV}$} & $\begin{array}{l}\text { CNEWSR } \\
\left(e^{2} \mathrm{fm}^{2}\right)\end{array}$ \\
\hline 0.40 & 92.7 & 75.0 & 93.5 & 87.0 & 72.8 & 1.77 \\
\hline 0.20 & 91.5 & 80.3 & 92.1 & 88.0 & 81.9 & 2.45 \\
\hline 0.10 & 90.1 & 82.9 & 90.5 & 87.9 & 87.9 & 3.32 \\
\hline 0.05 & 88.7 & 83.8 & 89.0 & 87.1 & 92.0 & 4.44 \\
\hline
\end{tabular}

the spatial extension of the bound ground state. Progressively weaker ground-state binding can require more components of continuum states in calculations of dipole excitations. This is also supported by the CNEWSR calculations presented in Table III. The percentage of a sum rule exhausted within the given energy ranges becomes smaller for more weakly bound ground states but in all cases it is larger than $80 \%$ and reaches more than $90 \%$ for the most bound case. Such a level of a convergence is quite reasonable for our exploratory calculations.

It is rather instructive to make comparisons of dipole strength calculations that take into account the final-state interactions (FSI) with calculations that neglect them, i.e., use the three-body plane waves (PW) as continuum wave functions. The PW calculations shown in Fig. 5 by the dashed lines isolate and clearly reveal the properties of dipole strength function that are connected to the bound ground state (like a Fourier transform). We see that the position of the dipole peak is mostly defined by the separation energy of the bound state and moves close to the threshold with decreasing binding. Inclusion of the FSI leads to redistribution of dipole transitions and forms more narrow and stronger bumps with only modest change of the peak position.

Figure 6 shows the ${ }^{22} \mathrm{C}$ dipole strength functions for bound ground states (described in Table I) with different two-neutron separation energies from 400 to $50 \mathrm{keV}$. The peak of the dipole strength function moves closer to the breakup threshold with decreasing separation energy and simultaneously rapidly grows in absolute value. To highlight this feature, the insert compares the strength function for separation energies 10 and $50 \mathrm{keV}$ (but note the different scales for two axes compared with the main figure). This remarkable enhancement clearly reflects the strong dependence of the soft dipole modes on the looseness of the halo systems and, respectively, on the difference between the charge and matter r.m.s. radii that increases with weakening of the binding.

The behavior of the dipole strength functions has a strong influence on EMD cross sections when the halo nucleus collides with the heavy target. An estimation of the Coulomb fragmentation can be obtained within the framework of firstorder perturbation theory [23,24]. According to the virtual photon method, the electromagnetic excitation of the halo nucleus can be described as absorption of virtual photons generated by the target nucleus and defined by the product of the dipole strength function and the spectrum of the virtual photons that peaks at low excitation energies. In this approximation the reaction mechanism depends on the minimum impact parameter, $b_{m}$. At impact parameter smaller than $b_{m}$, excitations are dominated by strong nuclear forces while at larger distances they are produced by electromagnetic interactions only and lead to Coulomb fragmentation. The minimum impact parameter $b_{m}$ depends on the considered process, but it is often taken equal to the sum of the two nuclear radii. In our estimation we used a target size equal to $1.2 A_{T}^{1 / 3}$ (where $A_{T}$ is the number of nucleons in the target) while for the halo size we applied the average distance of a halo neutron from the core c.m. (column $R_{C n}$ in Table I). Table IV gives cross sections for the ${ }^{22} \mathrm{C}$ Coulomb fragmentation (integrated up to $10 \mathrm{MeV}$ total continuum energy) caused by the collision with a ${ }^{208} \mathrm{~Pb}$ target at different collision energies. The cross section is rather sensitive to energy and, for each collision energy, to the two-nucleon separation energy of the ${ }^{22} \mathrm{C}$ nucleus. Especially remarkable are very small binding energies when the respective EMD cross sections may be very large. If the r.m.s matter radius (column $r_{m}$ in

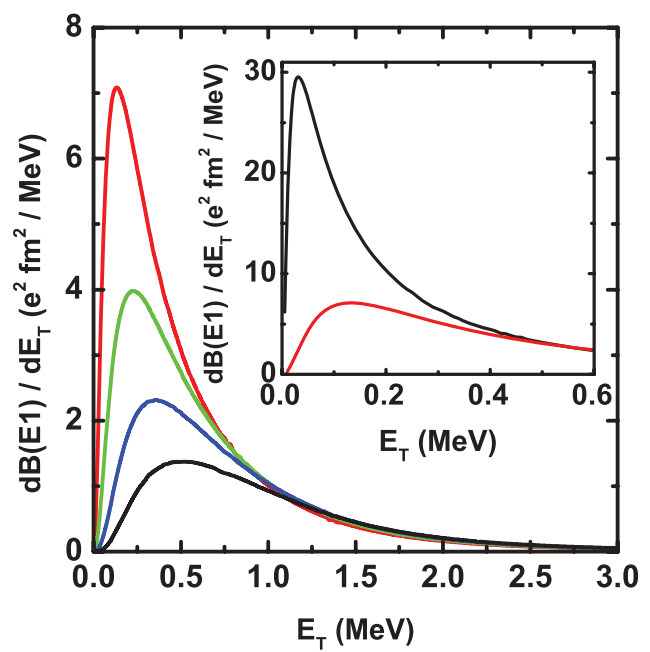

FIG. 6. (Color online) Calculated ${ }^{22} \mathrm{C}$ dipole strength function distributions for separation energies $S_{2 n}=50,100,200$, and $400 \mathrm{keV}$ (upper to lower curves). The insert compares dipole strength distributions for $S_{2 n}=10 \mathrm{keV}$ (upper line) and $50 \mathrm{keV}$ (lower line). 
TABLE IV. Estimation of the $E 1$ EMD cross sections $\sigma_{\mathrm{EMD}}$ (measured in barns) for the reaction ${ }^{22} \mathrm{C}+{ }^{208} \mathrm{~Pb}$ for different collision energies (per one nucleon). Estimations are given for different ${ }^{22} \mathrm{C}$ two-neutron separation energies $S_{2 n}$. The minimum impact parameters $b_{m}$ employed are also given.

\begin{tabular}{lcccrr}
\hline \hline$S_{2 n}(\mathrm{MeV})$ & 0.40 & 0.20 & 0.10 & 0.05 & 0.01 \\
$b_{m}(\mathrm{fm})$ & 13.5 & 14.6 & 16.0 & 17.5 & 21.2 \\
\hline$E / A(\mathrm{MeV})$ & $\sigma_{\text {EMD }}$ & $\sigma_{\text {EMD }}$ & $\sigma_{\text {EMD }}$ & $\sigma_{\text {EMD }}$ & $\sigma_{\text {EMD }}$ \\
\hline 240 & 1.2 & 1.8 & 2.7 & 3.9 & 8.1 \\
140 & 1.6 & 2.5 & 3.7 & 5.5 & 11.3 \\
40 & 3.2 & 5.4 & 8.5 & 12.9 & 28.3 \\
\hline \hline
\end{tabular}

Table I) is used for estimations of the minimum impact parameter instead of the distance between a halo neutron and core, $b_{m}$ become smaller and this leads to an enhanced EMD cross section by $10-20 \%$ depending on collision and separation energies.

\section{CONCLUSION}

Investigations of radioactive drip-line nuclei are now in the forefront of nuclear physics and spectacular progress has been made in this field. The breakthrough discovery of nuclear halos in some drip-line nuclei, in particular Borromean halos, is an important example. By now a substantial amount of information has been accumulated about the structure of such nuclei; cardinal examples have been ${ }^{6} \mathrm{He},{ }^{11} \mathrm{Li}$, and now recently ${ }^{22} \mathrm{C}$. These nuclei are characterized by having $p$-shell, mixed $s p$-shell, and $s$-shell structures, respectively. All halo nuclei are examples of weakly bound systems and their specific structure has an important influence on spatial extension. We may expect that if the $s$ motion plays the larger role in the nuclear structure, the respective system should have the bigger size. Thus it is to be expected that the ${ }^{22} \mathrm{C}$ size is increased more relatively to its core than what was observed for ${ }^{6} \mathrm{He}$ and ${ }^{11} \mathrm{Li}$ nuclei. However, the ${ }^{22} \mathrm{C}$ matter radius [1], extracted from experiment, if confirmed, is so large that the question about how it may be realized must be put forward. A possible answer certainly resides in the two-neutron separation energy $S_{2 n}$ of ${ }^{22} \mathrm{C}$, which is experimentally unknown.

In this article, we explored how the separation energy $S_{2 n}$ influences the spatial extension of an $s$-dominated Borromean halo nucleus and also other observables that are directly connected to the large size of the ground state. For that purposes we have applied the cluster three-body hyperspherical harmonic model that gives the most simple and physically transparent description of the two-neutron halo structure. To simulate the absence of a bound ${ }^{21} \mathrm{C}$ we used a shallow ${ }^{20} \mathrm{C}$-neutron potential that does not allow ${ }^{21} \mathrm{C}$ to bind, and we fixed its strength to the required binding energy of ${ }^{22} \mathrm{C}$.

These simulations show that, to reach the experimentally suggested lower boundary of the extracted matter radius, the separation energy $S_{2 n}$ of two neutrons in ${ }^{22} \mathrm{C}$ should be of the order of tens of $\mathrm{keV}$, while to reach the mean value the binding must be much weaker. Within the cluster model the matter extension of the nucleus is directly connected to the distance between the matter and charge c.m. positions. This leads to a swelling of the ${ }^{22} \mathrm{C}$ pointlike charge radius due to the core motion about the nuclear c.m., compared with the original charge size of the ${ }^{20} \mathrm{C}$ core. Also, the strength and position of soft dipole excitations are very sensitive to the separation energy and thus to the system's spatial extension. The position of the soft dipole peak moves closer to the three-body threshold with decreasing separation energy $S_{2 n}$. Correspondingly, the separation between the average position of matter and charge distributions becomes larger and the height of the dipole peak is increased in accordance with the non-energy-weighted dipole sum rule. Since the flux of virtual photons peaks at small excitation energies, this results in EMD cross sections that are very sensitive to the separation energy and can reach rather big values for the weak binding. Experimental excitation (breakup) measurements may clarify this open question, and these are urgently called for.

\section{ACKNOWLEDGMENT}

SNE acknowledges financial support from the University of Bergen.
[1] K. Tanaka et al., Phys. Rev. Lett. 104, 062701 (2010).

[2] G. Audi, A. H. Wapstra, and C. Thibault, Nucl. Phys. A 729, 337 (2003).

[3] M. T. Yamashita et al., Phys. Lett. B 697, 90 (2011); 715, 282(E) (2012).

[4] H. T. Fortune and R. Sherr, Phys. Rev. C 85, 027303 (2012).

[5] M. V. Zhukov et al., Phys. Rep. 231, 151 (1993).

[6] M. V. Zhukov et al., Phys. Lett. B 265, 19 (1991).

[7] J. Raynal and J. Revai, Nuovo Cim. A 68, 612 (1970).

[8] W. Horiuchi and Y. Suzuki, Phys. Rev. C 74, 034311 (2006).

[9] K. Varga and Y. Suzuki, Phys. Rev. C 52, 2885 (1995).

[10] Y. Suzuki and K. Varga, in Stochastic Variational Approach to Quantum-Mechanical Few-Body Problems, Lecture Notes in Physics, Vol. m54 (Springer-Verlag, Berlin, 1998).

[11] G. E. Brown and A. D. Jackson, Nucleon-Nucleon Interactions (North Holland, Amsterdam, 1976).

[12] A. Ozawa et al., Nucl. Phys. A 691, 599 (2001).
[13] B. V. Danilin, S. N. Ershov, and J. S. Vaagen, Phys. Rev. C 71, 057301 (2005).

[14] W. Horiuchi and Y. Suzuki, Phys. Rev. C 76, 024311 (2007).

[15] W. Horiuchi, Y. Suzuki, B. Abu-Ibrahim, and A. Kohama, Phys. Rev. C 75, 044607 (2007).

[16] P. G. Hansen and B. Jonson, Europhys. Lett. 4, 409 (1987).

[17] T. Kobayashi et al., Phys. Lett. B 232, 51 (1989).

[18] K. Ikeda, INS Report JHP-7, 1988 (in Japanese).

[19] B. V. Danilin et al., Nucl. Phys. A 632, 383 (1998).

[20] S. N. Ershov, B. V. Danilin, and J. S. Vaagen, Phys. Rev. C 64, 064609 (2001).

[21] C. Forssen, V. D. Efros, and M. V. Zhukov, Nucl. Phys. A 697, 639 (2002).

[22] Y. Alhassid, M. Gai, and G. F. Bertsch, Phys. Rev. Lett. 49, 1482 (1982).

[23] A. Winther and K. Alder, Nucl. Phys. A 319, 518 (1979).

[24] C. A. Bertulani and G. Baur, Nucl. Phys. A 442, 739 (1985). 